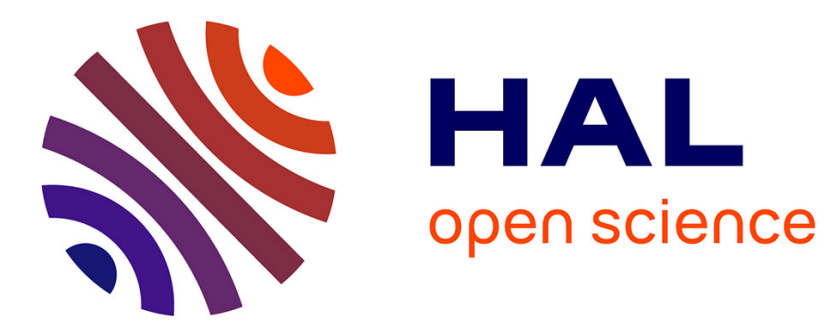

\title{
Navigation and Guidance Strategy Planning for UAV Urban Operation
}

\author{
Yoko Watanabe, Aurélien Veillard, Caroline Ponzoni Carvalho Chanel
}

\section{To cite this version:}

Yoko Watanabe, Aurélien Veillard, Caroline Ponzoni Carvalho Chanel. Navigation and Guidance Strategy Planning for UAV Urban Operation. AIAA Science and Technology Forum and Exposition Forum (SciTech 2016), Jan 2016, San Diego, United States. pp. 1-15. hal-01503685

\section{HAL Id: hal-01503685 \\ https://hal.science/hal-01503685}

Submitted on 7 Apr 2017

HAL is a multi-disciplinary open access archive for the deposit and dissemination of scientific research documents, whether they are published or not. The documents may come from teaching and research institutions in France or abroad, or from public or private research centers.
L'archive ouverte pluridisciplinaire HAL, est destinée au dépôt et à la diffusion de documents scientifiques de niveau recherche, publiés ou non, émanant des établissements d'enseignement et de recherche français ou étrangers, des laboratoires publics ou privés. 


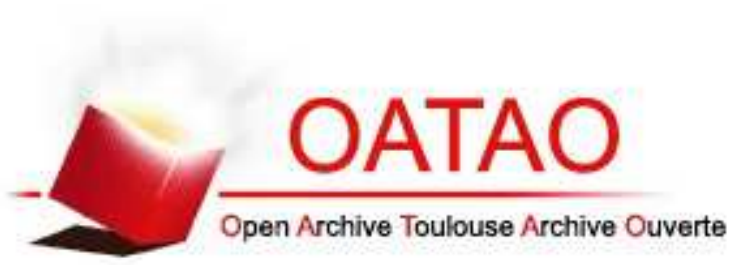

\section{Open Archive TOULOUSE Archive Ouverte (OATAO)}

OATAO is an open access repository that collects the work of Toulouse researchers and makes it freely available over the web where possible.

This is an author-deposited version published in : http://oatao.univ-toulouse.fr/ Eprints ID : 15091

To cite this version : Watanabe, Yoko and Veillard, Aurélien and Ponzoni Carvalho Chanel, Caroline Navigation and Guidance Strategy Planning for UAV Urban Operation. (2016)

In: Proceedings of AIAA Science and Technology Forum and Exposition Forum (SciTech 2016), 4 January 2016 - 8 January 2016 (San Diego, United States).

Any correspondance concerning this service should be sent to the repository administrator: staff-oatao@listes-diff.inp-toulouse.fr 


\title{
Navigation and Guidance Strategy Planning for UAV Urban Operation
}

\author{
Yoko Watanabe*, Aurélien Veillard ${ }^{\dagger}$ \\ ONERA - The French Aerospace Laboratory \\ 2 av. Edouard Belin - BP74025 - 31055 TOULOUSE Cedex 4 - FRANCE \\ Caroline P. Carvalho Chanel ${ }^{\ddagger}$ \\ ISAE-SUPAERO, Université de Toulouse \\ 10 av. Edouard Belin - BP 54032 - 31055 TOULOUSE Cedex 4 - FRANCE
}

\begin{abstract}
This paper proposes a concept of navigation and guidance strategy planner for urban operation of a VTOL-type UAV. One of major challenges of UAV autonomous navigation in an urban environment is to deal with the risk of GPS signal occlusion. In order to address this issue, various approaches have been proposed for GPS-independent UAV navigation and guidance such as visual odometry and visual servoing control. In this context, this work supposes that different navigation and guidance modes using different set of sensors are available onboard an UAV. An idea of the proposed planner is to anticipate the navigation and guidance performance degradation (or amelioration) due to unavailability (or availability) of certain mode in the path planning task. The planning problem is formulated as a 5D (3D position + selection of navigation and guidance modes) graph search problem, where the localization and path execution uncertainties are propagated according to a model of corresponding modes for each node transition. Node transition is denied if the path execution uncertainty ellipsoid intercects with any obstacle. A minimizing cost function is defined by a volume of the path execution uncertainty corridor, as it implies minimizing path distance and execution accuracy at the same time. A deterministic graph search algorithm is applied to find a flight path with specified navigation and guidance mode transitions which minimizes the defined cost function. Simulations are performed by using path planning configurations given in an existing UAV obstacle field navigation benchmark, and the results are presented to prove the proposed navigation and guidance strategy planning concept.
\end{abstract}

\section{Introduction}

In recent years, there are increasing demands of unmanned aerial vehicles (UAVs) performing a mission, either civil or military, in a remote complex environment; inspection of infrastructure such as railway or pipeline, scientific observation and mapping (archeology, agriculture, etc.), disaster relief and recovery, and reconnaissance \& surveillance in an adversarial environment. In order to reduce workload and eligibility requirements for UAV operators in such mission operations, an UAV onboard flight system is required to have a maximum level of autonomy to ensure its safety in a complex environment.

One of the challenges associated with UAV autonomous operation in an urban (or even rurban) environment is to make its navigation and guidance system robust to GPS signal loss/degradation due to occlusion. Onboard flight system on most outdoor UAVs highly relies on the precision of GPS localization. The INS-only navigation solution diverges very quickly due to accumulation of inertial measurement bias, and consequently, UAV loses its automatic flight capability in the absence of GPS signal. In order to address this issue, one

\footnotetext{
*Research engineer, Systems Control and Flight Dynamics Department, Yoko.Watanabe@onera.fr

${ }^{\dagger}$ Internship student, Systems Control and Flight Dynamics Department, Aurelien.Veillard@onera.fr

${ }^{\ddagger}$ Research engineer, Complex Systems Engineering Department, Caroline.Chanel@isae-supaero.fr
} 
can find intensive research work on development of alternative navigation and guidance systems which do not rely on GPS information. For example, a variety of visual odometry and SLAM (Simultaneous Localization and Mapping) techniques have been proposed to estimate UAV state by either using purely vision or by fusing vision with INS (Inertial Navigation System) and/or other available sensors ( ${ }^{1-3}$ and many others). Visual servoing approaches can be applied to UAV guidance and control relative to its environment without using UAV absolute state. For example, UAV terrain following, corridor navigation or landing can be achieved by using vision information directly in a flight controller ${ }^{4-} \cdot{ }^{6}$ Such systems give an important key to realize an UAV autonomous navigation in absence of GPS signals. However, their localization or guidance and control performance is often degraded compared to that of a nominal flight system using GPS information.

In this context, the work presented in this paper originates in the idea of anticipating GPS signal occlusion risk and related flight performance degradation in UAV path planning task. Methods to predict GPS availability and accuracy at a given location can be found in literature. For example, methods proposed in $^{7}$ and $^{8}$ use multipath effect risk and a skyview angle, respectively, which are calculated from a $3 \mathrm{D}$ model of the surrounding area. A GPS availability map obtained through such methods can be very useful in path planning to choose a safe flight path which ensures no collision risk under the UAV localization uncertainty possibly degraded due to GPS unavailability.

Path planning under localization uncertainty is not new in the robotics community. Especially, a large amount of work has been done for navigating a ground mobile robot which possesses two different navigation modes; dead-reckoning and landmark-based localization $^{9-} .^{12}$ These works apply a deterministic or a sampling-based search algorithm to find a minimum-distance collision risk-free path while evolving a vehicle self-localization error ellipsoid. A choice among the two navigation modes are imposed in function of landmark visibility, and is not included as a planning object. One of the authors of this paper presented a safe path planner under localization uncertainty for UAV urban operation. ${ }^{13}$ Unlike the work listed above, the proposed planner in ${ }^{13}$ not only plans a flight path but also a navigation strategy (i.e., a choice of localization mode to be used on each path segment) by taking into account GPS unavailable zones and the UAV self-localization performance degradation in such zones.

This paper is an extension of this previous work by adding different guidance modes available onboad an UAV. In the previous work, the UAV localization uncertainty is considered to coincide with the UAV path execution accuracy. It is reasonable when assuming an absolute guidance law, such as waypoint tracking, which regulates the UAV localization. However in reality, it is unlikely to fly an UAV in a proximity of obstacles without any relative sensing and navigation function. Therefore, in addition to the UAV localization modes, this paper supposes different guidance modes with their availabilities and considers a closed-loop path execution uncertainty rather than the localization uncertainty. As a simple example, two guidance modes are given in this paper for a VTOL-type UAV; waypoint tracking (absolute guidance) and vision-based wall-following (relative guidance). The flight path execution uncertainty is modeled differently for each mode. An interesting point here is that the path execution uncertainty does not depend on the UAV absolute localization error when applying the wall following guidance. In other words, this relative guidance mode enables to ensure UAV flight safety regarding to collision risk even with a large degradation in the UAV self-localization accuracy due to GPS signal loss. Similarly to GPS or other sensors, availability of this wall-following guidance mode is limited and it depends on the surrounding environment (i.e. existance and proximity of a wall).

This paper first states a problem of this navigation and guidance strategy planning (Section II), then provides a propagation model of UAV self-localization and path execution uncertainties for each navigation and guidance mode (Section III, IV). Section V describes how the uncertainty propagation is incorporated in path planning task. Section ?? presents simulation results of the proposed guidance and navigation strategy planner. Section ?? includes concluding remarks and future perspectives. 


\section{Problem Statement}

An objective of our path planner is to find the shortest collision risk-free path from a start point $X_{\text {start }}$ to a goal $X_{\text {goal }}$ in a $3 D$ cluttered environment. The UAV onboard system is supposed to have several different navigation (i.e., localization) modes as well as guidance modes, which give different evolution of the localization and path execution error covariance. Therefore, the planner is also required to select the best combination of the navigation and guidance modes on each segment of the planned path. The planner assumes the following a-priori knowledge :

- Environment Map : a 3D occupancy map, each of whose voxel contains a binary value (free/occupied). It can be built from 3D point cloud obtained by vision or lidar scan data.

- Obstacle classification : a label of obstacles (for example, tree or building) for each occupied voxel in the 3D occupancy map. This information will be used to identify an availability of a certain guidance mode (e.g. wall following).

- Sensor Availability Maps : 3D voxel maps of the same size as the environment map, each of whose voxel contains a binary value (available/not). It can be constructed by considering characteristics of each sensor and its environment (e.g. GPS signal occlusion, visibility of landmark, wall sensing range).

- Localization Uncertainty Models : EKF (Extended Kalman Filter) process is applied for UAV self-localization. It includes a prediction step with INS measurements and an update step with a selected sensor measurement (specified for each navigation mode). This EKF-based model is used to propagate the localization error covariance along the planned path from its given initial value $P_{\text {start }}$.

- Path Execution Uncertainty Models : a closed-loop translational dynamics model with a selected guidance mode is used to propagate the path execution error covariance along the planned path. Each guidance mode uses different information (UAV selflocalization result, sensor measurement, etc.), and hence transfers errors in the used information into the path execution error.

- Minimum Safety Distance : minimum separation distances from obstacles in each of the UAV body axis. It should be a sum of the UAV size and safety margin.

The collision risk is identified if separation distance between the path execution error ellipsoid and occupied voxels is less than the minimum safety distance.

\section{UAV Navigation Modes}

This section details the UAV self-localization systems which are used to propagate the localization error covariance from one node to another in the path planner. This paper considers the following four different means of localization.

1) INS-only : Integration of INS measurements only. It is always available, but diverges very quickly.

2) GPS/INS : Integration of INS measurements with GPS position and velocity measurement update. It gives precise localization, but not available in GPS signal occluded area.

3) Optical flow/INS : Integration of INS measurements with optical flow field measurement update. It can limit a divergence of the INS-only localization, but not available over non-flat surface.

4) Landmark/INS : Integration of INS measurements with landmark pixel coordinate measurement update. Visual landmark detection algorithm is assumed to be available, and the landmark position is known a-priori. It is available only when a landmark is in the field of view of the onboard camera.

An EKF estimation process is applied to each of those self-localization systems.

\section{A. Integration of INS Acceleration}

Let $X, V$ and $a$ be the UAV position, velocity and acceleration in the locally-fixed inertial reference frame (North-East-Up frame). Assuming the precise attitude estimation, the 
INS acceleration measurement is modeled by

$$
\boldsymbol{a}_{I N S}=L_{B I} \boldsymbol{a}+\Delta \boldsymbol{a}+\boldsymbol{w}_{a}
$$

where $L_{B I}$ is a direction cosine matrix from the reference frame to the UAV body frame. $\Delta a$ is an additive acceleration bias and $w_{a}$ is a measurement noise. Let

$$
\boldsymbol{x}=\left[\begin{array}{lll}
\boldsymbol{X}^{T} & \boldsymbol{V}^{T} & \Delta \boldsymbol{a}^{T}
\end{array}\right]^{T}
$$

be the estimation state vector. Then its dynamics can be given by

$$
\dot{\boldsymbol{x}}=\left[\begin{array}{c}
\dot{\boldsymbol{X}} \\
\dot{\boldsymbol{V}} \\
\dot{\Delta} \boldsymbol{a}
\end{array}\right]=\left[\begin{array}{c}
\boldsymbol{V} \\
\boldsymbol{a} \\
\boldsymbol{w}_{\Delta a}
\end{array}\right]=F \boldsymbol{x}+G \boldsymbol{a}_{I N S}+G_{w} \boldsymbol{w}
$$

where $\boldsymbol{w}=\left[\begin{array}{ll}\boldsymbol{w}_{a}^{T} & \boldsymbol{w}_{\Delta a}^{T}\end{array}\right]^{T}$ is a Gaussian process noise and

$$
F=\left[\begin{array}{ccc}
O & I & O \\
O & O & -L_{B I}^{T} \\
O & O & O
\end{array}\right], \quad G=\left[\begin{array}{c}
O \\
L_{B I}^{T} \\
O
\end{array}\right], \quad G_{w}=\left[\begin{array}{cc}
O & O \\
-L_{B I}^{T} & O \\
O & I
\end{array}\right]
$$

Given an estimate $\hat{\boldsymbol{x}}_{k-1}$, its error covariance $P_{k-1}$ at time $t_{k-1}$ and the INS acceleration input at time $t_{k}$, the estimation state and its error covariance are propagated to time $t_{k}$ according to

$$
\begin{aligned}
\hat{\boldsymbol{x}}_{k}^{1} & =\Phi \hat{\boldsymbol{x}}_{k-1}+G \boldsymbol{a}_{I N S_{k}} \Delta t_{k} \\
P_{k}^{1} & =\Phi P_{k-1} \Phi^{T}+G_{w} Q G_{w}^{T} \Delta t_{k}
\end{aligned}
$$

where $\Phi=I+F \Delta t_{k}, \Delta t_{k}=t_{k}-t_{k-1}$ is the state transition matrix, $Q$ is a covariance matrix of the process noise $w$.

\section{B. Sensor Measurement Updates}

When a measurement of one of the sensors is available, the INS-propagated estimation $\hat{\boldsymbol{x}}_{k}^{1}$ is corrected through EKF update process. Let

$$
\boldsymbol{z}_{k}^{m}=\boldsymbol{h}_{m}\left(\boldsymbol{x}\left(t_{k}\right)\right)+\boldsymbol{\nu}_{k}^{m}
$$

be a measurement obtained by $m$-th sensor at time $t_{k}$. Then, the update process becomes

$$
\begin{aligned}
\hat{\boldsymbol{x}}_{k}^{m} & =\hat{\boldsymbol{x}}_{k}^{1}+K_{k}^{m}\left(\boldsymbol{z}_{k}^{m}-\boldsymbol{h}_{m}\left(\hat{\boldsymbol{x}}_{k}^{1}\right)\right) \\
P_{k}^{m} & =\left(I-K_{k}^{m} H_{k}^{m}\right) P_{k}^{1}
\end{aligned}
$$

where $K_{k}^{m}=P_{k}^{1} H_{k}^{m T}\left(H_{k}^{m} P_{k}^{1} H_{k}^{m T}+R_{k}^{m}\right)^{-1}$ is a Kalman gain, $H_{k}^{m}$ is a Jacobian matrix of the measurement model $\boldsymbol{h}_{m}\left(\hat{\boldsymbol{x}}_{k}^{1}\right)$ and $R_{k}^{m}$ is a covariance matrix of the measurement noise $\boldsymbol{\nu}_{k}^{m}$. The measurement models for the four different sensors listed at the beginning of the section are summarized below;

- GPS : The absolute position and velocity measurement, $\boldsymbol{h}_{2}(\boldsymbol{x})=\left[\begin{array}{ll}\boldsymbol{X}^{T} & \boldsymbol{V}^{T}\end{array}\right]^{T}$.

- Optical flow : Suppose that a camera is fixed on a UAV at a position $X_{c}^{B}$ in the UAV body frame looking nadir. Then the camera position and velocity become

$$
\begin{aligned}
\boldsymbol{X}_{c} & =\boldsymbol{X}+L_{B I}^{T} \boldsymbol{X}_{c}^{B} \\
\boldsymbol{V}_{c} & =\boldsymbol{V}+L_{B I}^{T}\left(\boldsymbol{\omega}_{B} \times \boldsymbol{X}_{c}^{B}\right)
\end{aligned}
$$

where $\omega_{B}$ is UAV angular velocity in the body frame. When UAV pitch and roll angles are small, the optical flow field on a flat ground surface $Z=Z_{0}$ can be approximated by the following affine model.

$$
\dot{\boldsymbol{p}}=\left[\begin{array}{cc}
w_{c} & r_{c} \\
-r_{c} & w_{c}
\end{array}\right] \boldsymbol{p}-f\left[\begin{array}{l}
u_{c}+q_{c} \\
v_{c}-p_{c}
\end{array}\right]=A \boldsymbol{p}+\boldsymbol{b}
$$

where $\boldsymbol{p}$ is a pixel coordinates, $f$ is a focal length in pixel, and

$$
\boldsymbol{\omega}^{C}=L_{C B} \boldsymbol{\omega}^{B}=\left[\begin{array}{c}
p_{c} \\
q_{c} \\
r_{c}
\end{array}\right], \quad \boldsymbol{v}^{C}=\frac{L_{C I} \boldsymbol{V}_{c}}{Z_{0}-Z_{c}}=\left[\begin{array}{c}
u_{c} \\
v_{c} \\
w_{c}
\end{array}\right]
$$


Suppose that the UAV system has an image processor which estimates the affine model of the optical flow field $(A, b) .{ }^{14}$ Then we can extract the measurement of

$$
\boldsymbol{h}_{3}(\boldsymbol{x})=\boldsymbol{v}^{C}(\boldsymbol{x})=\frac{L_{C I} \boldsymbol{V}+L_{C B}\left(\boldsymbol{\omega}_{B} \times \boldsymbol{X}_{c}^{B}\right)}{Z_{0}-Z-\boldsymbol{e}_{3}^{T} L_{B I}^{T} \boldsymbol{X}_{c}^{B}}
$$

where $Z_{0}$ is a ground elevation provided by the environment model.

- Landmark : Let $X_{l}$ be a position of a landmark in the reference frame, which is known a-priori to the UAV system. If this landmark is inside the field of view of the onboard camera, a landmark detection algorithm can measure its pixel coordinates;

$$
\boldsymbol{h}_{4}(\boldsymbol{x})=\boldsymbol{p}_{l}(\boldsymbol{x})=\frac{f}{Z_{l}^{C}}\left[\begin{array}{l}
X_{l}^{C} \\
Y_{l}^{C}
\end{array}\right], \quad \boldsymbol{X}_{l}^{C}=L_{C I}\left(\boldsymbol{X}_{l}-\boldsymbol{X}_{c}\right)
$$

where the camera position $X_{c}$ given by (5).

\section{UAV Guidance Modes}

This section describes the UAV guidance laws which are used to propagate the path execution error covariance from one node to another in the path planner. This paper considers the following two different guidance modes.

1) Waypoint tracking : Path following by using the UAV self-localization result (Section III). This mode is available everywhere, but its path execution accuracy directly depends on the UAV localization accuracy.

2) Wall following : Path following relative to a wall by using onboard vision-based sensor measurement but not the UAV localization result. It uses the optical flow and range measurements, which give relative velocity information to the detected wall, directly in the guidance law. This mode is available in a given sensing range from voxels labeled "wall", when the path direction is close to parallel to the wall orientation.

\section{A. Waypoint Tracking Guidance}

Consider a transition from one voxel $X_{k-1}$ in the 3D occupancy map to one of its 26 neighboring voxels $X_{k}$. Let $u$ be an unit vector from $X_{k-1}$ to $X_{k}$, and $V_{0}$ is a desired cruise speed. Then, a reference trajectory for this node transit can be defined by

$$
\begin{aligned}
& \boldsymbol{X}^{*}(t)=\boldsymbol{X}_{k-1}+V_{0}\left(t-t_{k-1}\right) \boldsymbol{u} \\
& \boldsymbol{V}^{*}(t)=V_{0} \boldsymbol{u}
\end{aligned}
$$

for $t_{k-1} \leq t \leq t_{k}$ with $t=t_{k}$ as a time at the initial node $X_{k-1} \cdot t_{k}$ is a time at the destination node $\boldsymbol{X}_{k}$, and defined by $t_{k}=t_{k-1}+\left\|\boldsymbol{X}_{2}-\boldsymbol{X}_{1}\right\| / V_{0}=t_{k-1}+\Delta t_{k}$. Let

$$
\boldsymbol{e}=\left[\begin{array}{ll}
\left(\boldsymbol{X}-\boldsymbol{X}^{*}\right)^{T} & \left(\boldsymbol{V}-\boldsymbol{V}^{*}\right)^{T}
\end{array}\right]^{T}
$$

be a reference trajectory tracking error. Then its dynamics can be written by

$$
\dot{\boldsymbol{e}}=\left[\begin{array}{cc}
O & I \\
O & O
\end{array}\right] \boldsymbol{e}+\left[\begin{array}{c}
O \\
I
\end{array}\right](\boldsymbol{a}+\boldsymbol{w})=A \boldsymbol{e}+B(\boldsymbol{a}+\boldsymbol{w})
$$

where $w$ is a process noise whose covariance matrix is given by $Q$. By assuming a small deviation in the initial condition, a simple linear guidance law can be applied to follow this reference trajectory.

$$
\boldsymbol{a}=-K_{p}\left(\hat{\boldsymbol{X}}(t)-\boldsymbol{X}^{*}(t)\right)-K_{d}\left(\hat{\boldsymbol{V}}(t)-\boldsymbol{V}^{*}(t)\right)
$$

where $\hat{\boldsymbol{X}}$ and $\hat{\boldsymbol{V}}$ are the UAV absolute position and velocity estimation results from Section III. Then the closed-loop trajectory tracking error becomes

$$
\dot{\boldsymbol{e}}=\left[\begin{array}{cc}
O & I \\
-K_{p} & -K_{d}
\end{array}\right] \boldsymbol{e}+\left[\begin{array}{ccc}
O & O & O \\
K_{p} & K_{d} & O
\end{array}\right] \tilde{\boldsymbol{x}}+\left[\begin{array}{c}
O \\
I
\end{array}\right] \boldsymbol{w}=\tilde{A} \boldsymbol{e}+\tilde{B} \tilde{\boldsymbol{x}}+B \boldsymbol{w}
$$

where $\tilde{\boldsymbol{x}}$ is the estimation error of the state defined in (1). By integrating this dynamic equation from $t_{k-1}$ to $t_{k}$, the trajectory tracking error at time $t_{k}$ is given by

$$
\boldsymbol{e}\left(t_{k}\right)=e^{\tilde{A} \Delta t_{k}} \boldsymbol{e}\left(t_{k-1}\right)+\int_{t_{k-1}}^{t_{k}} e^{\tilde{A}\left(t_{k}-s\right)} \tilde{B} \tilde{\boldsymbol{x}}(s) d s+\int_{t_{k-1}}^{t_{k}} e^{\tilde{A}\left(t_{k}-s\right)} B \boldsymbol{w}(s) d s
$$




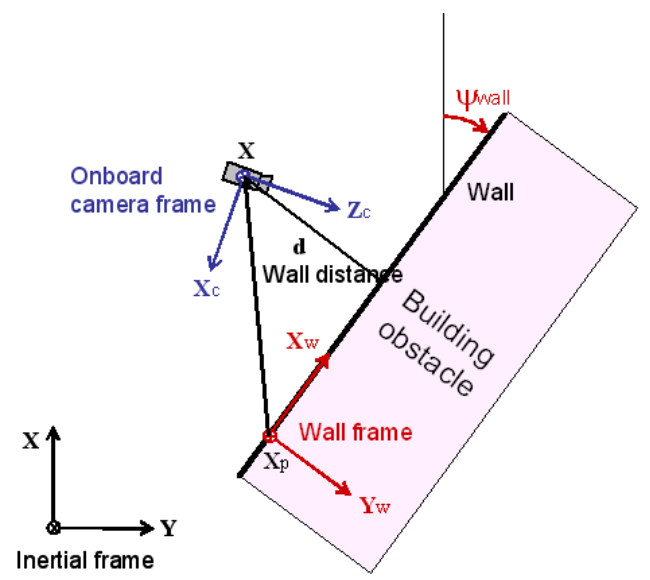

Figure 1: Wall Frame

Finally, the trajectory tracking error covariance, denoted by $E^{1}\left(t_{k}\right)$, can be calculated as follows.

$$
\begin{aligned}
E^{1}\left(t_{k}\right) & =\mathbb{E}\left[\boldsymbol{e}\left(t_{k}\right) \boldsymbol{e}^{T}\left(t_{k}\right)\right] \\
& =e^{\tilde{A} \Delta t_{k}} E\left(t_{k-1}\right) e^{\tilde{A}^{T} \Delta t_{k}}+\int_{t_{k-1}}^{t_{k}} e^{\tilde{A}\left(t_{k}-s\right)} \tilde{B} P(s) \tilde{B}^{T} e^{\tilde{A}^{T}\left(t_{k}-s\right)} d s+\int_{0}^{t} e^{\tilde{A}(t-s)} B Q B^{T} e^{\tilde{A}^{T}(t-s)} d s
\end{aligned}
$$

where $E\left(t_{k-1}\right)$ is the initial trajectory tracking error covariance, $P(t)$ is the UAV selflocalization error covariance resulting from the EKF process in Section III. Assuming a small time interval, we can approximate an evolution of the trajectory tracking error covariance from time $t_{k-1}$ to $t_{k}$ by

$$
E_{k}^{1}=E_{k-1}+\left(\tilde{A} E_{k-1}+E_{k-1} \tilde{A}^{T}+\tilde{B} P_{k-1} \tilde{B}^{T}+B Q B^{T}\right) \Delta t_{k}
$$

The tracking error covariance at the destination node $X_{k}$ is given by this $E_{k}^{1}$.

\section{B. Wall Following Guidance}

The second guidance mode consists in a wall following by using optical flow and range measurements, but without using the UAV absolute localization results. Suppose a vertical wall whose orientation is $\psi_{\text {wall }}$ in the $3 \mathrm{D}$ world. We consider the same node transition and the same reference trajectory as in the previous subsection, assuming that the both nodes are within the proximity sensing range from the wall. Taking a fixed point on the wall $X_{p}$ as an origin, a wall-fixed frame is defined by

$$
\boldsymbol{X}^{W}=L_{3}\left(\psi_{\text {wall }}\right)\left(\boldsymbol{X}-\boldsymbol{X}_{p}\right)
$$

where $L_{3}$ represents $Z$-axis rotation matrix. The wall distance $d$ is defined by $d=-Y^{W}$, a negative of the second component of this $X^{W}$. Figure 1 illustrates this wall frame. The reference trajectory (7-8) is transformed to this wall frame as follows.

$$
\begin{aligned}
& \boldsymbol{X}^{* W}(t)=\boldsymbol{X}_{k-1}^{W}+V_{0} t L_{3}\left(\psi_{\text {wall }}\right) \boldsymbol{u}=\boldsymbol{X}_{k-1}^{W}+V_{0} t \boldsymbol{u}^{W} \\
& \boldsymbol{V}^{* W}(t)=V_{0} L_{3}\left(\psi_{\text {wall }}\right) \boldsymbol{u}=V_{0} \boldsymbol{u}^{W}
\end{aligned}
$$

According to ${ }^{4}$ from the optical flow measurement, one can deduce the information $v^{C}=$ $V^{C} / d$, the UAV velocity in the onboard camera frame scaled by the wall distance. Besides, the wall distance $d$ can be measured by a range sensor. Assuming a perfect knowledge on the camera attitude (hence a rotation matrix from the camera frame to the wall frame $\left.L_{W C}\right)$, the following measurement can be used in the reference trajectory tracking guidance.

$$
\begin{aligned}
\hat{Y}^{W} & =Y^{W}-\tilde{Y}^{W}=-\hat{d} \\
\hat{\boldsymbol{V}}^{W} & =\boldsymbol{V}^{W}-\tilde{\boldsymbol{V}}^{W}=\hat{d} L_{W C} \hat{\boldsymbol{v}}^{C}
\end{aligned}
$$


where $\tilde{\boldsymbol{V}}^{W}$ and $\tilde{Y}^{W}$ are the measurement errors.

$$
\begin{aligned}
\tilde{Y}^{W} & =-\tilde{d} \\
\tilde{\boldsymbol{V}}^{W} & \simeq \hat{d} L_{W C} \tilde{\boldsymbol{v}}^{C}+\tilde{d} L_{W C} \hat{\boldsymbol{v}}^{C}
\end{aligned}
$$

The wall following guidance law can be designed in a similar manner as the waypoint tracking guidance law;

$$
\boldsymbol{a}^{W}=-K_{p}\left(\hat{\boldsymbol{X}}^{W}(t)-\boldsymbol{X}^{* W}(t)\right)-K_{d}\left(\hat{\boldsymbol{V}}^{W}(t)-\boldsymbol{V}^{* W}(t)\right)
$$

But as we do not have a position information parallel to the wall, there is no position error feedback on $X^{W}$ and $Z^{W}$ axes and it results in the linear gain in a form of

$$
K_{p}=\left[\begin{array}{ccc}
0 & 0 & 0 \\
0 & k_{p} & 0 \\
0 & 0 & 0
\end{array}\right]
$$

The trajectory tracking error dynamics becomes

$$
\left.\dot{\boldsymbol{e}}^{W}=\left[\begin{array}{cc}
O & I \\
-K_{p} & -K_{d}
\end{array}\right] \boldsymbol{e}^{W}+\left[\begin{array}{c}
\mathbf{0} \\
0 \\
k_{p} \\
0
\end{array}\right] \quad \begin{array}{c}
O \\
K_{d}
\end{array}\right]\left[\begin{array}{c}
\tilde{Y}^{W} \\
\tilde{\boldsymbol{V}}^{W}
\end{array}\right]+\left[\begin{array}{c}
O \\
I
\end{array}\right] \boldsymbol{w}^{W}=\tilde{A} \boldsymbol{e}^{W}+\tilde{B}\left[\begin{array}{c}
\tilde{Y}^{W} \\
\tilde{\boldsymbol{V}}^{W}
\end{array}\right]+B \boldsymbol{w}^{W}
$$

From the similar procedure as in Section IV.A, the trajectory tracking error covariance in the wall frame can be propagated from $t_{k-1}$ to $t_{k}$ by

$$
E_{k}^{2 W}=E_{k-1}^{W}+\left(\tilde{A} E_{k-1}^{W}+E_{k-1}^{W} \tilde{A}^{T}+\tilde{B} R_{k-1} \tilde{B}^{T}+B Q^{W} B^{T}\right) \Delta t_{k}
$$

$R_{k}$ is a measurement error covariance matrix of (15-16) given by

$$
R_{k} \simeq H_{k}\left[\begin{array}{cc}
r_{d} & \mathbf{0}^{T} \\
\mathbf{0} & R_{O F}
\end{array}\right] H_{k}^{T}, \quad H_{k}=\left[\begin{array}{cc}
-1 & \mathbf{0}^{T} \\
L_{W C} \hat{\boldsymbol{v}}^{C} & \hat{d} L_{W C}
\end{array}\right]
$$

where $r_{d}$ and $R_{O F}$ are the range and optical flow sensor error covariances respectively. This covariance matrix can be easily converted from the wall frame to the inertial frame by applying a rotation matrix $L_{3}\left(-\psi_{\text {wall }}\right)$. We recall here that, unlike a case of the waypoint tracking guidance, the uncertainty in the trajectory tracking error (19) does not depend on the UAV self-localization uncertainty.

\section{Path Planning}

The main idea of this paper is to apply a graph search algorithm to find a safe and short path under the path execution uncertainty $E$ which evolves differently with a combination from the four different UAV self-localization modes presented in Section III and the two different guidance modes presented in Section IV.

\section{A. Environment Model}

In this work, a 3D voxel model is used to represent the environment as a graph. Let $\Delta X$ be a resolution and $X_{0}$ be a position of the most South-West-Bottom corner node of the model. Then, a position corresponding to a $(i, j, k)$-voxel is

$$
\boldsymbol{X}_{(i, j, k)}=\boldsymbol{X}_{0}+\left[\begin{array}{ccc}
i-1 & 0 & 0 \\
0 & j-1 & 0 \\
0 & 0 & k-1
\end{array}\right] \Delta \boldsymbol{X}
$$

An occupancy of the $(i, j, k)$-voxel is a-priori known. Furthermore, each occupied voxel has an obstacle label which identifies if it corresponds to a building-type obstacle where the wall following guidance mode is applicable or not. This object classification can be done by using a method proposed in $^{15}$ for example, and walls can be detected as contours of the building-type obstacles. The $3 \mathrm{D}$ voxel model is given by

$$
\operatorname{map}\left(\boldsymbol{X}_{(i, j, k)}\right)= \begin{cases}2 & \text { if occupied and wall } \\ 1 & \text { if occupied but not wall } \\ 0 & \text { if free }\end{cases}
$$


On the graph, a neighborhood of the $(i, j, k)$-voxel is defined by its 26 adjacent ones; $(i+$ $\delta i, j+\delta j, k+\delta k)$ where $\delta i, \delta j, \delta k \in\{-1,0,1\}$. In addition to the occupancy map, an availability map for each of the four navigation modes and of the two guidance modes is supposed to be created from the $3 \mathrm{D}$ voxel model $\operatorname{map}\left(X_{(i, j, k)}\right)$. An availability of the $m$-th navigation mode at the $(i, j, k)$-voxel is given by

$$
\operatorname{navmap}\left(\boldsymbol{X}_{(i, j, k)}, m\right)= \begin{cases}1 & \text { if the } m \text {-th navigation mode is available } \\ 0 & \text { if the } m \text {-th navigation mode is unavailable }\end{cases}
$$

Similarly, an availability of the $l$-th guidance mode at the $(i, j, k)$-voxel is given by

$$
\operatorname{guimap}\left(\boldsymbol{X}_{(i, j, k)}, l\right)= \begin{cases}1 & \text { if the } l \text {-th guidance mode is available } \\ 0 & \text { if the } l \text {-th guidance mode is unavailable }\end{cases}
$$

As states in Section III and IV, the navigation and guidance mode availability condition is given by sensor characteristics used in each mode. Table 1 summarizes the availability condition for each mode. GPS availability condition is simplified with a sky-view angle threshold in this work. However, it is desirable to use a more precise GPS availability model such as those found in $^{7}$ and. ${ }^{8}$

\begin{tabular}{|c|c|c|c|}
\hline \multicolumn{3}{|c|}{ Modes } & Availability Condition \\
\hline \multirow{4}{*}{ 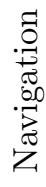 } & 1 & INS-only & Everwhere \\
\hline & 2 & GPS & Sky-view angle $>$ threshold \\
\hline & 3 & Optical flow & Elevation variance of a surface appeared on the image $<$ threshold \\
\hline & 4 & Landmark & Landmark is within the onboard camera field-of-view \\
\hline \multirow{3}{*}{ } & 1 & Waypoint & Everywhere \\
\hline & 2 & Wall & Distance to a wall $<$ sensing range, and \\
\hline & & & Angle between path direction and wall $<$ threshold \\
\hline
\end{tabular}

Table 1: Availability Condition of Each Navigation and Guidance Modes

\section{B. Uncertainty Propagation Model}

Consider a UAV displacement from a voxel $X_{0}$ to its neighbor $X_{1}$. Suppose the UAV localization and path execution error covariance matrices at $\boldsymbol{X}_{0}$ are given by $P_{0}$ and $E_{0}$ respectively. If navmap $\left(\boldsymbol{X}_{1}, m\right)=1$, the localization error covariance can be propagated to $P_{1}^{m}$ through the corresponding observation model in Section III. If guimap $\left(\boldsymbol{X}_{1}, l\right)=1$, the path execution error covariance can be propagated to $E_{1}^{l}$ through the corresponding model in Section IV, which may use the UAV localization error covariance $P_{0}$.

\section{Posability and Traversability Conditions}

Let $E_{X}>0$ be the position tracking error covariance. In this paper, an error ellipsoid is defined by the conventional $2 \sigma$-error ellipsoid of $E_{X}$ augmented by the safety distance $d_{s a f e}$. Assuming the zero mean error $\hat{X}=X_{0}$, the UAV is posable at the voxel $X_{0}$ with the path execution error covariance $E_{0}$ if $\operatorname{map}(X)=0$ for ${ }^{\forall} \boldsymbol{X}$ within the augmented ellipsoid of $E_{X 0}$. Now we consider a traversability from a voxel $X_{0}$ with the path execution error covariance $E_{0}$ to a voxel $X_{1}$ with the updated covariance $E_{1}$. The first condition of the traversability is that the UAV must be posable at the both initial and destinate positions. Then, one should check if there is a collision between the evolving error ellipsoid and occupied voxels along the edge connecting the two. For calculation simplicity, the evolution of the error ellipsoid is approximated by a truncated elliptic cone shown in Figure 2 in this work. The UAV is traversable from $X_{0}$ with $E_{0}$ to $X_{1}$ with $E_{1}$, if $\operatorname{map}(X)=0$ for all nodes within this elliptic cone.

\section{Uncertainty Corridor}

In many existing work of path planning under uncertainty, a minimum distance (or time, energy) path is calculated with a traversability constraint on the error ellipsoid. Some 


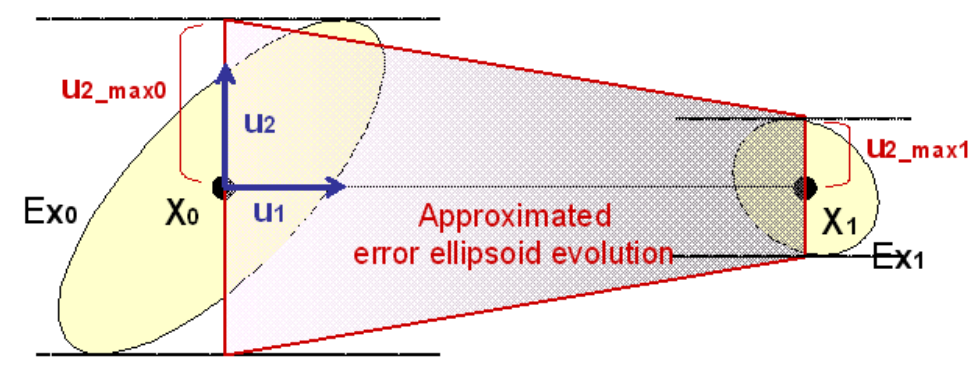

Figure 2: Elliptic Cone Approximation of the Error Ellipsoid Evolution between Two Voxels

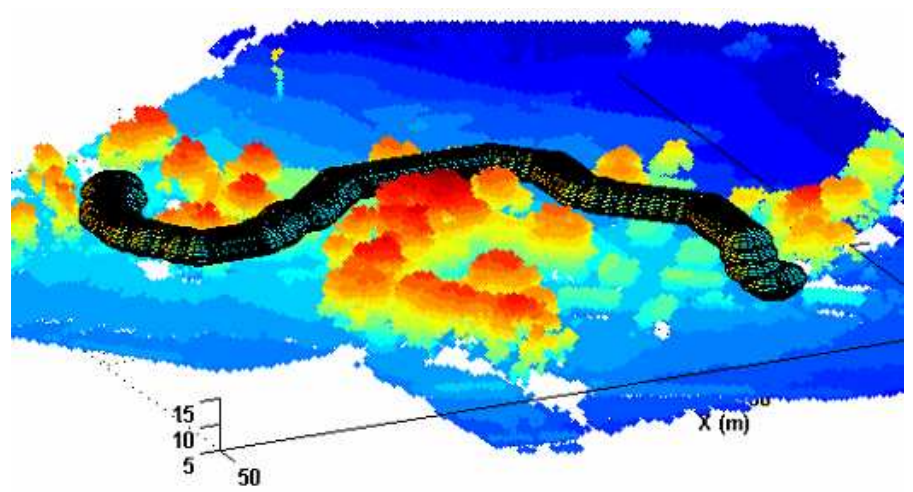

Figure 3: Example of Uncertainty Corridor

propose a cost function as a weighed sum of the distance and a localization uncertainty cost (e.g. ${ }^{16}$ ) to integrate the uncertainty minimization in the planning objective. However, such a cost function requires an ad-hoc parametrization to decide how much priority to be put on minimum uncertainty over minimum distance. This paper inherits a concept of uncertainty corridor proposed in. ${ }^{13}$ The uncertainty corridor is defined by a space swept by the path execution error ellipsoid $E$ evolving along a planned path from the start to the goal. Figure 3 shows an example of the uncertainty corridor. As done in, ${ }^{13}$ a volume of this uncertainty corridor is chosen as a cost function in the planning task. Minimizing the volume of the uncertainty corridor implies minimizing both the path length and the accumulated path execution uncertainty. The uncertainty corridor can be approximated by an ensemble of the elliptic cones (Figure 2) between two successive voxels on the path. Therefore, the cost function is given by

$$
\begin{aligned}
J= & \frac{\pi}{6} \sum_{n=2}^{N}\left\|\boldsymbol{X}_{\left(i_{n}, j_{n}, k_{n}\right)}-\boldsymbol{X}_{\left(i_{n-1}, j_{n-1}, k_{n-1}\right)}\right\| \cdot\left(u_{2_{\max n}} u_{3_{\max }-1}+\right. \\
& \left.u_{2_{\max _{n-1}}} u_{3_{\max _{n}}}+2\left(u_{2_{\max _{n-1}}} u_{3_{\max _{n-1}}}+u_{2_{\max _{n}}} u_{3_{\max n}}\right)\right)
\end{aligned}
$$

where $\left\{\boldsymbol{X}_{1}, \boldsymbol{X}_{2}, \cdots, \boldsymbol{X}_{N}\right\}$ constitutes a planned path.

\section{E. Path Search Algorithms}

In order to find a collision risk-free path, which minimizes a cost function defined in (20), one can apply a classical graph search algorithm such as $A^{*} .^{17}$ In this work, a graph is built in a 5D search space where a node corresponds to a combination of a $3 \mathrm{D}$ voxel of the environment map $\boldsymbol{X}_{(i, j, k)}$, a navigation mode $m$ and a guidance mode $l$. One node has maximum 208 (26 neighbouring voxels $\times 4$ navigation modes $\times 2$ guidance modes) connections. A connection to a node $\left(\boldsymbol{X}_{(i, j, k)}, m, l\right)$ is established if the $m$-th navigation and $l$-th guidance modes are available at the voxel $X_{(i, j, k)}$, and the edge is "traversable" with the corresponding evolution of the path execution uncertainty. $A^{*}$ search algorithm can be applied with the following cost and heuristic functions. 
- cScore : a transition cost from one node to another. A volume of the approximated uncertainty corridor (the truncated elliptic cone) is used.

- hScore : a heuristic function estimating a cost-to-go at the current node to the goal. An infimum limit of the volume of the uncertainty corridor from the current node to the goal, corresponding to the case of direct connection with $E_{X}=O$, is used.

- gScore : a cost to reach to the current node from the start, a summation of cScores of edges on the path.

- fScore : an estimated total cost, a sum of the gScore and hScore. This is used as a priority queue in the algorithm.

Differences from the conventional minimum-distance $A^{*}$ search are; i) it optimizes the cost (distance + uncertainty) over selections of a path along with the navigation and guidance modes, ii) the traversability condition under the uncertainty is imposed to confirm a connection between two nodes, iii) it outputs not only a sequence of nodes $\left(\boldsymbol{X}_{\text {path }}\right)$ but also a sequence of the navigation and guidance modes $\left(M_{p a t h}, L_{p a t h}\right)$ associated with each path segment.

\section{F. Post Processing}

A resulting path $X_{\text {path }}$ is represented as a sequence of adjacent voxels in the $3 \mathrm{D}$ occupancy map. In order to execute the planned path, it needs to be converted to a more simplified flight plan which can be given by a sequence of sparse waypoints. In order to eliminate intermediate voxels on a given path $\boldsymbol{X}_{\text {path }}$, Theta*-like process is performed on it. Theta* algorithm is an any-angle path planner proposed in. ${ }^{18}$ It adds to $A^{*}$ algorithm an extra process of checking the connectivity from a parent of the current node to a neighbored node before checking a direct connectivity from the current node to the neighbor. If the neighbored node can be connected to the parent, it establishes this connection by omitting the current node. The similar process is applied to omit the intermediate points on the path, by checking the traversability and the availability of navigation and guidance mode specified at each node.

\section{Simulation Results}

The concept of the navigation and guidance strategy planner proposed in this paper has been validated through simulations with obstacle configurations defined in the UAV obstacle field navigation benchmark. ${ }^{19}$ This benchmark is open to public, and obstacle models and baseline trajectory data are available online. It contains six different simple obstacle configurations, and a terrain model of San Diego downtown for real urban environment. This paper uses four of the simple obstacle configurations.

\section{A. Simulation Settings}

This subsection describes simulation and path planning settings used in our simumlation validation.

- Path Planning Configurations: Four different configurations shown in Figure 4 are used. Every obstacles have $20 \mathrm{~m}$ of height above a ground surface. Start and goal points are separated by $100 \mathrm{~m}$, both at the altitude $10 \mathrm{~m}$ above ground.

$$
\boldsymbol{X}_{\text {start }}=\left[\begin{array}{c}
0 \\
0 \\
10
\end{array}\right](m), \quad \boldsymbol{X}_{\text {goal }}=\left[\begin{array}{c}
0 \\
100 \\
10
\end{array}\right](m)
$$

The minimum safety distance is set as $8 m$ in horizontal axes and $6 m$ in vertical. The nominal UAV speed is given by $3.5 \mathrm{~m} / \mathrm{s}$. For simplification, the estimation of the accleration bias $\Delta a$ is omitted. The initial UAV self-localization error covariance is given by

$$
P_{\text {start }}=\left[\begin{array}{cll}
(2)^{2} I & \left(m^{2}\right) & O \\
O & (0.4)^{2} I & \left((\mathrm{~m} / \mathrm{sec})^{2}\right)
\end{array}\right]
$$

The inital path execution error covariance $E_{\text {start }}$ coincides with $P_{\text {start }}$. 


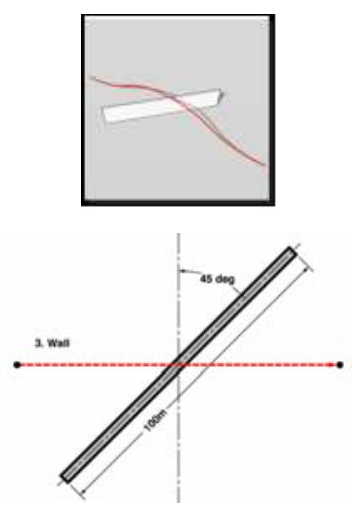

BM3) Wall
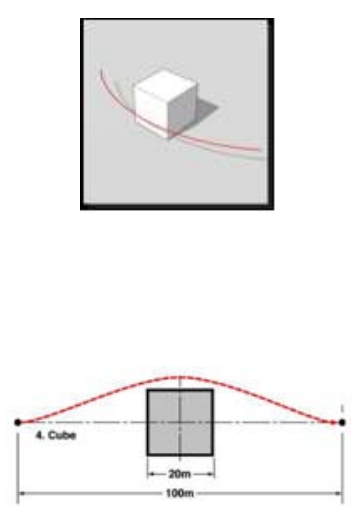

BM4) Cube

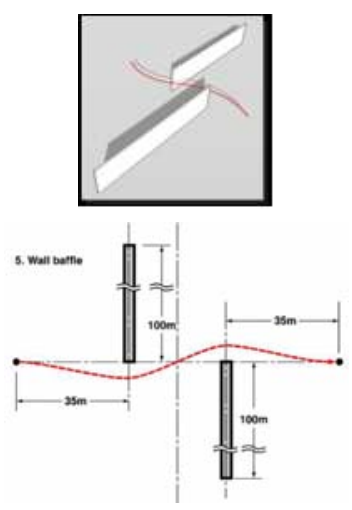

BM5) Wall Baffle

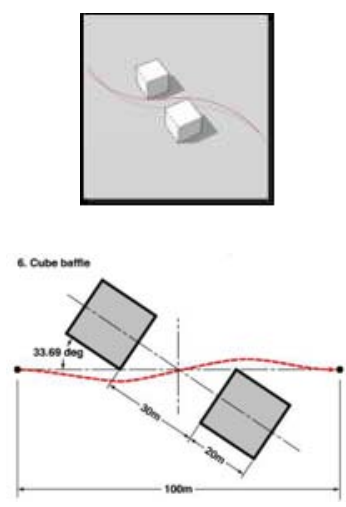

BM6) Cube Baffle

Figure 4: Obstacle Configurations ${ }^{19}$

- Environment map: Each obstacle configuration is represented as a 3D-voxel occupancy map with $2 m$ of resolution in horizontal and $3 m$ in vertical. The map covers from $\left[\begin{array}{lll}-100 & -50 & 0\end{array}\right]^{T}(m)$ to $\left[\begin{array}{lll}100 & 150 & 40\end{array}\right]^{T}(m)$. It means that the map contains $101 \times 101 \times$ 14 voxels. All obstacles are considered as "building" and their contours corresnpond to "wall" to which the wall-following guidance mode is applicable.

- Navigation and guidance mode availability map: For each voxel on the 3D occupancy map, availability of each navigation and guidance mode is calculated according to the conditions in Table 1. $90^{\circ}$ is used for the sky-view angle threshold for the GPS availability. The onboard camera is supposed to have $60^{\circ}$ field-of-view for each axis. As the tested environment has parfectly flat ground surface, the elevation variance threshold for the optical flow-based navigation mode is set to 0 . For the wall-following guidance mode, $15 \mathrm{~m}$ wall sensing range and $\pm 30^{\circ}$ angle threshold are used.

- Parameters in the navigation modes: The following covariance matrices for the process noise and the measurement noise are used in the EKF process.

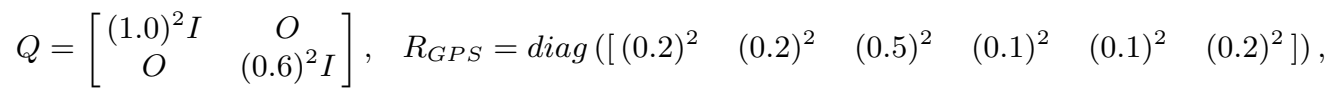

$$
\begin{aligned}
& R_{O F}=\operatorname{diag}\left(\left[\begin{array}{lll}
(0.02)^{2} & (0.02)^{2} & (0.04)^{2}
\end{array}\right]\right), \quad R_{L M}=(0.03)^{2} I
\end{aligned}
$$

The EKF prediction and update process is iterated with a sampling time 0.1 sec, except the GPS update process with $0.5 \mathrm{sec}$, on each path segment.

- Parameters in the guidance modes: The linear control gain is set as $k_{p}=0.1$ and $k_{d}=1.4 \sqrt{k_{p}}$, which gives a damping ratio $\xi=0.7$ and a natural frequency $\omega_{n}=\sqrt{0.1}$ in the closed-loop system. The measurement noise covariance of the optical flow is the same as that in the optical flow-based navigation mode, and that of the distance measurement is $(0.1)^{2}$. The process noise is set to zero.

\section{B. Results}

The following four cases are tested in simulations.

- TEST1: Most outdoor UAVs rely on the GPS/INS self-localization and use waypoints to navigate. If GPS signal is lost, they have no choice but to use the INS-only localization mode. In the first simulation set, we assume only the INS-only and GPS/INS navigation modes and the waypoint tracking guidance mode available onboard an UAV.

- TEST2: One of the solutions to navigate an UAV in a partially GPS-denied environment is to prepare an alternative GPS-free navigation system. In this second simulation set, the optical flow-based navigation system is added to the TEST1 case.

- TEST3: In the third set, instead of adding the vision-based navigation system, the vision-based wall-following guidance system is added to the TEST1 case.

- TEST4: In the last simulation set, all the four navigation modes and the two guidance modes are supposed to be available onbaord. 


Config.

Figure 5: TEST1: Strategy Planning Results with Nominal System

\section{Conclusion}

This paper proposed an UAV navigation and guidance strategy planner which takes into account an evolution of the path execution uncertainty, in function of availability and choice of different UAV self-localization and guidance modes, in order to ensure the flight safety. In our previous paper, ${ }^{13}$ we have shown that the UAV navigation strategy planner can be effective in planning a safer flight trajectory under GPS signal occlusion risk. This paper extended this work by considering path execution uncertainty, rather than localization uncertainty, with different guidance modes. While some guidance mode rely on the UAV localization result, the others do not. The concept of this navigation and guidance strategy planning is validated through simulations.

The work of this paper applied a classical graph search algorithm to find the optimal strategy and flight plan to reach a given goal. However, this approach has some limitations. For instance, the path execution uncertainty is used for the traversability check and the cost calculation. But it is not really treated as a probability distribution of the node transition (i.e. UAV displacement). That is, with such path execution uncertainty, the node transition should include more than one possibilities with corresponding probabilities for an applied guidance law. Hence, the planner needs to deal with this "uncertain" node transition associated with the path execution uncertainty. Moreover, we would like to include an "observation" process in the strategy plannig. During a mission execution, the 


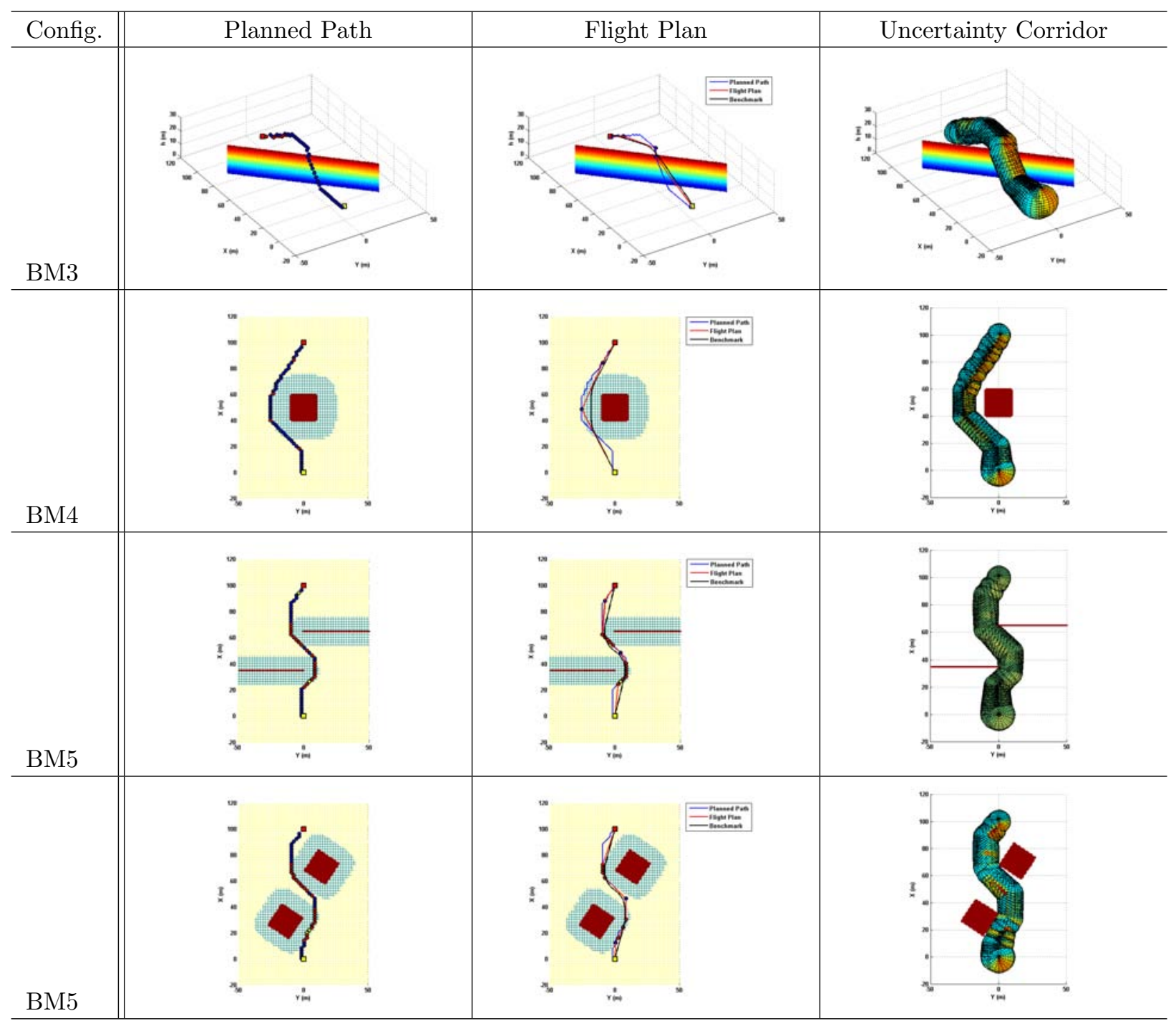

Figure 6: TEST2: Strategy Planning Results with Vision-based Navigation System

UAV onboars system is capable to detect a current availability of each of the navigation and guidance mode (e.g. GPS signal reception, landmark or wall detection). Therefore, the planner must adapt a strategy in function of this observation result. In order to treate those two aspects, the authors are currently working on formulating the poblem in POMDP (Partially Observable Markov Decision Process) framework ${ }^{20} .{ }^{21}$ POMDP handles decisional problems where the result of actions applied to the system are uncertain, and where the observations of discrete states are partial or imprecise.

Finally, our future perspective includes development of an online embeddable strategy planner. As our problem dimension is larger than that of conventional flight path planners, it is a big challenge for us to develop an online planner which runs onboard an UAV during the mission execution flight in order to replan a strategy upon new environment and UAV state perception, and upon new observation of sensor availability.

\section{References}

${ }^{1}$ G. Chowdhary, E.N. Johnson, D. Magree, A. Wu and A. Shein. GPS-denied Indoor and Outdoor Monocular Vision Aided Navigation and Control of Unmanned Aircraft. Journal of Field Robotics, 2013.

${ }^{2}$ M. Sanfourche, V. Vittori and G. le Besnerais. Evo: A Realtime Embedded Stereo Odometry 


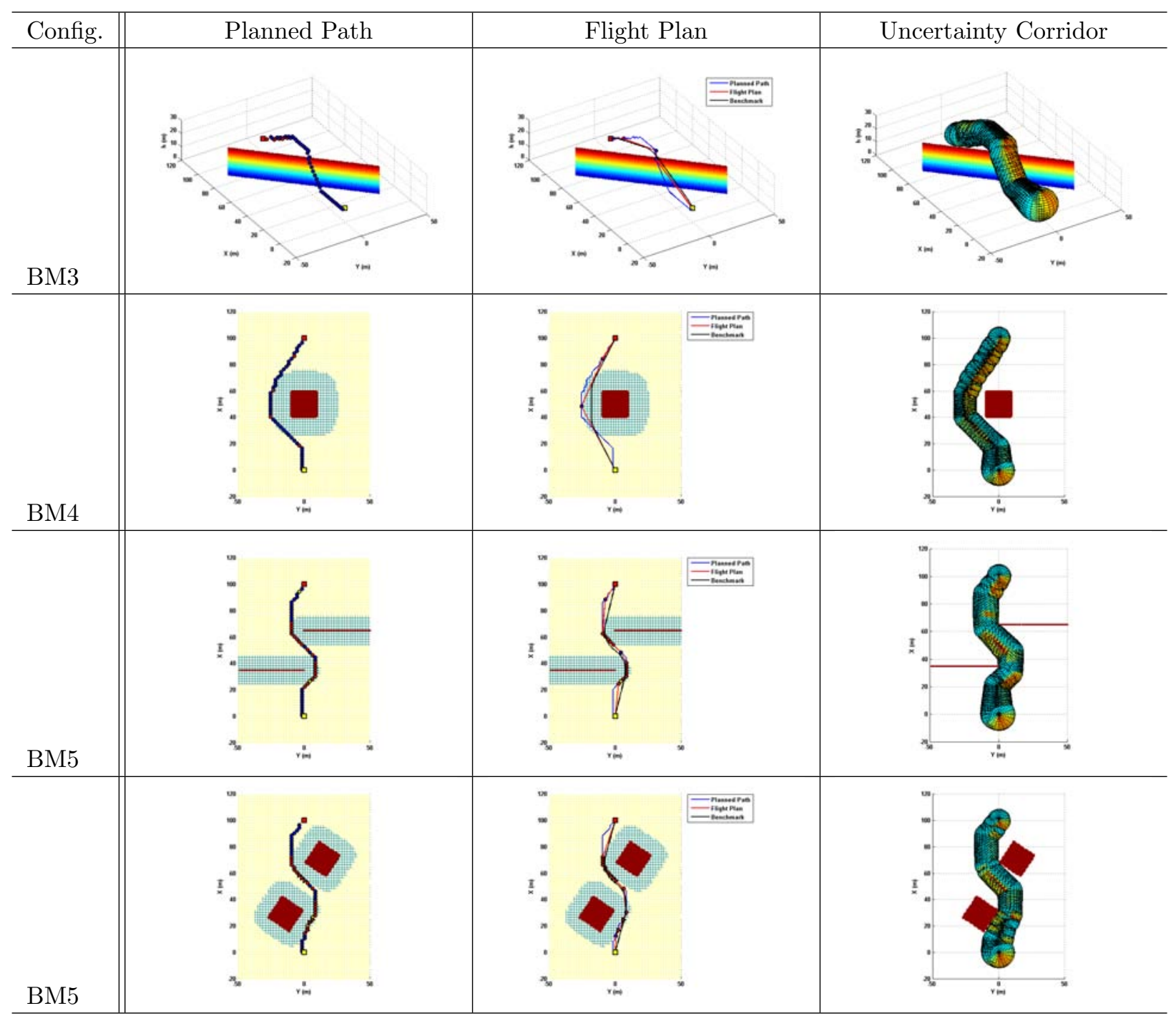

Figure 7: TEST4: Strategy Planning Results with Vision-based Navigation and Guidance System

for MAV Applications. IEEE International Conference on Intelligent Robots and Systems, 2013.

${ }_{3}^{3}$ S. Lynen, S. Omari, M. Wuest, M. Achtelik and R. Siegwart. Tightly Coupled Visual-Inertial Navigation System Using Optical Flow. IFAC Workshop on Research, Education and Development of Unmanned Aerial Systems, 2013.

${ }^{4}$ B. Herisse, S. Oustrieres, T. Hamel, R. Mahony and F.-X. Russotto. A General Optical Flow based Terrain Following Strategy for a VTOL UAV using Multi Views. IEEE International Conference on Robotics and Automation, 2010.

${ }^{5}$ G. Chowdhary, D.M. Sobers, E. Salaun, J. Ottander and E.N. Johnson. Fully Autonomous Indoor Flight Relying on Only Five Very Low-Cost Range Sensors. Journal of Aerospace Information Systems, 2013.

${ }^{6}$ S. Zingg, D. Scaramuzza, S. Weiss and R. Siegwart. MAV Navigation through Indoor Corridors Using Optical Flow. IEEE International Conference on Robotics and Automation, 2010.

${ }^{7}$ F. Kleijer, D. Odijk and E. Verbree. Prediction of GNSS Availability and Accuracy in Urban Environments Case Study Schiphol Airport. Location Based Services and TeleCartography II - Lecture Notes in Geoinformation and Cartography, Springer Berlin Heidelberg, 2009.

${ }^{8}$ B.J. Beeseley. Sky Viewshed Modeling for GPS Use in the Urban Environment. M.S. Thesis, University of South Carolina, 2002.

${ }^{9}$ R. Alami and T. Simeon. Planning Robust Motion Strategies for Mobile Robot. IEEE International Conference on Robotics and Automation, 1994.

${ }^{10}$ A. Lambert and D. Gruyer. Safe Path Planning in an Uncertain-Configuration Space. IEEE International Conference on Robotics and Automation, 2003.

${ }^{11}$ R. Pepy and A. Lambert. Safe Path Planning in an Uncertain-Configuration Space using RRT. IEEE/RSJ International Conference on Intelligent Robot and Systems, 2006. 
${ }^{12}$ S. Candido and S. Hutchinson. Minimum Uncertainty Robot Path Planning using a POMDP Approach. IEEE/RSJ International Conference on Intelligent Robot and Systems, 2010.

${ }^{13}$ Y. Watanabe, S. Dessus and P. Fabiani. Safe Path Planning with Localization Uncertainty for Urban Operation of VTOl UAV. AHS Annual Forum, 2014.

${ }^{14}$ Y. Watanabe, P. Fabiani and G. Le Besnerais. Simultaneous Visual Target Tracking and Navigation in a GPS-denied Environment. International Conference on Advanced Robotics, 2009.

${ }^{15}$ B. Le Saux and M. Sanfourche. Rapid Semantic Mapping: Learn Environment Classifiers on the Fly. IEEE/RSJ International Conference on Intelligent Robot and Systems, 2013.

${ }^{16}$ B.D. Luders, S. Karaman and J.P. How. Robust Sampling-based Motion Planning with Asymptotic Optimality Guarantees. AIAA Guidance, Navigation and Control Conference, 2013.

${ }^{17}$ P. Hart, N. Nilsson and B. Raphael. A Formal Basis for the Heuristic Determination of Minimum Cost Paths. IEEE Transactions of Systems Science and Cybernetics, 1968.

${ }^{18}$ A. Nash, K. Daniel, S. Koenig and A. Felner, Theta*: Any-Angle Path Planning on Grids. AAAI Conference on Artificial Intelligence, 2007.

${ }^{19}$ B. Mettler, C. Goerzen, Z. Kong and M. Whalley. Benchmarking of Obstacle Field Navigation Algorithms for Autonomous Helicopters. AHS Annual Forum, 2010.

${ }^{20}$ R.D. Smallwood and E.J. Sondik. The Optimal Control of Partially Observable Markov Processes over a Finite Horizon. Operations Research, Informs, 1973.

${ }^{21}$ L.P. Kaelbling, M.L. Littman and A.R. Cassandra. Planning and Acting in Partially Observable Stochastic Domains. Artificial intelligence, Elsevier, 1998. 